International Journal of Pure and Applied Mathematics

Volume 84 No. 4 2013, 365-370

ISSN: 1311-8080 (printed version); ISSN: 1314-3395 (on-line version)

url: http://www.ijpam.eu

doi: http://dx.doi.org/10.12732/ijpam.v84i4.6

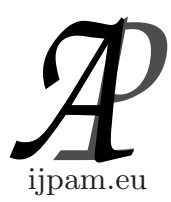

\title{
AN IMPROVED BOUND ON GEOMETRIC APPROXIMATION BY $w$-FUNCTIONS
}

\author{
K. Teerapabolarn \\ Department of Mathematics \\ Faculty of Science \\ Burapha University \\ Chonburi, 20131, THAILAND
}

Abstract: In this paper, we improve a result of geometric approximation in
$[2]$ to be more appropriate for any $q \in(0,1)$.

AMS Subject Classification: 62E17, 60F05

Key Words: geometric approximation, non-uniform bound, stein's method, $w$-functions

\section{Introduction}

Let $X$ be a non-negative integer-valued random variable with probability function $p(x)>0$ for every $x$ in the support of $X, \mathcal{S}(x)$. For $x_{0} \in \mathcal{S}(x)$, let $\mathbb{F}\left(x_{0}\right)=\sum_{x=0}^{x_{0}} p(x)$ denote the distribution function of $X$ at $x_{0}$ and $\mu$ and $\sigma^{2}$ $\left(0<\sigma^{2}<\infty\right)$ denote the mean and variance of $X$, respectively. It is well known that the distribution of $X$ can be approximated by some discrete distributions if their parameters are satisfied under certain conditions. Let $\mathbb{G}_{p}\left(x_{0}\right)=\sum_{x=0}^{x_{0}} p q^{x}$ denote the geometric distribution function with parameter $p=1-q$ at $x_{0}$. If

Received: November 9, 2012

(c) 2013 Academic Publications, Ltd. url: www.acadpubl.eu 
we expect the distribution of $X$ to be closer to the geometric distribution than other distributions, then it is reasonable to estimate $\mathbb{F}\left(x_{0}\right)$ by $\mathbb{G}_{p}\left(x_{0}\right)$. In this case, Teerapabolarn [2] gave uniform and non-uniform bounds for the difference $\left|\mathbb{F}\left(x_{0}\right)-\mathbb{G}_{p}\left(x_{0}\right)\right|$ for $x_{0} \in \mathbb{N} \cup\{0\}$ and $\mu=\frac{q}{p}$ as follows:

$$
\left|\mathbb{F}\left(x_{0}\right)-\mathbb{G}_{p}\left(x_{0}\right)\right| \leq \sum_{x \in \mathcal{S}(x)}\left|q-\frac{\sigma^{2} w(x) p}{x+1}\right| p(x)
$$

and if $0<q \leq \frac{1}{2}$ then

$$
\left|\mathbb{F}\left(x_{0}\right)-\mathbb{G}_{p}\left(x_{0}\right)\right| \leq \frac{1}{x_{0}+1} \sum_{x \in \mathcal{S}(x)}\left|(x+1) q-\sigma^{2} w(x) p\right| p(x),
$$

where $w$ is a function associated with the non-negative integer-valued random variable $X$ defined as follows:

$$
w(x)=\frac{1}{\sigma^{2}}\left\{\mu+\frac{\sigma^{2} w(x-1) p(x-1)}{p(x)}-x\right\}, x \in \mathcal{S}(x) \backslash\{0\}
$$

and $w(0)=\frac{\mu}{\sigma^{2}}$. It is observed that the bound in (1.2) can be applied for $q \in\left(0, \frac{1}{2}\right]$. In this study, we are interest to improve this bound to be more appropriate for any $q \in(0,1)$.

\section{Method}

We will improve our main result by using the same methodology as in [2], which consists of Stein's method and $w$-functions. For $w$-functions, it follows from Majsnerowska [1] that mentioned in (1.3). For Stein's method, following [2], Stein's equation of the geometric distribution function with parameter $p \in(0,1)$ is of the form

$$
h_{x_{0}}(x)-\mathbb{G}_{p}\left(x_{0}\right)=q(1+x) g_{x_{0}}(x+1)-x g_{x_{0}}(x),
$$

where $x_{0}, x \in \mathbb{N} \cup\{0\}, h_{x_{0}}(x)=1$ if $x \leq x_{0}$ and $h_{x_{0}}(x)=0$ if $x>x_{0}$ and

$$
g_{x_{0}}(x)= \begin{cases}\frac{1}{x} \sum_{k=0}^{x-1} q^{k} \sum_{j=x_{0}+1}^{\infty} p q^{j-x} & \text { if } x \leq x_{0}, \\ \frac{1}{x} \sum_{k=0}^{x_{0}} q^{k} & \text { if } x>x_{0} \\ 0 & \text { if } x=0 .\end{cases}
$$


Let $\Delta g_{x_{0}}(x)=g_{x_{0}}(x+1)-g_{x_{0}}(x)$. Then, by $(2.2)$ and for $x \geq 1$, we have that

$$
\Delta g_{x_{0}}(x)= \begin{cases}\sum_{k=x_{0}+1}^{\infty} q^{k}\left[\frac{1}{(x+1) q^{x+1}} \sum_{j=0}^{x} p q^{j}-\frac{1}{x q^{x}} \sum_{j=0}^{x-1} p q^{j}\right] & \text { if } x \leq x_{0}, \\ \sum_{k=0}^{x_{0}} q^{k}\left[-\frac{1}{x(x+1)}\right] & \text { if } x>x_{0} .\end{cases}
$$

Lemma 2.1. For $x, x_{0} \in \mathbb{N}, \Delta g_{x_{0}}(x+1)-\Delta g_{x_{0}}(x)>0$ for every $x \leq x_{0}-1$.

Proof. Let $\Delta^{2} g_{x_{0}}(x)=\Delta g_{x_{0}}(x+1)-\Delta g_{x_{0}}(x)$. From (2.3), we have

$$
\begin{aligned}
\Delta^{2} g_{x_{0}}(x)= & \sum_{k=x_{0}+1}^{\infty} q^{k}\left\{\left[\frac{1}{(x+2) q^{x+2}} \sum_{j=0}^{x+1} p q^{j}-\frac{1}{(x+1) q^{x+1}} \sum_{j=0}^{x} p q^{j}\right]\right. \\
& \left.-\left[\frac{1}{(x+1) q^{x+1}} \sum_{j=0}^{x} p q^{j}-\frac{1}{x q^{x}} \sum_{j=0}^{x-1} p q^{j}\right\rfloor\right\} \\
= & \sum_{k=x_{0}+1}^{\infty} q^{k}\left\{\frac{(x+1) p-\left(q-q^{x+2}\right)}{(x+1)(x+2) q^{x+2}}-\frac{x p-\left(q-q^{x+1}\right)}{x(x+1) q^{x+1}}\right\} \\
= & \sum_{k=x_{0}+1}^{\infty} q^{k}\left\{\frac{x^{2} p^{2}+x p^{2}-2 x p q+2 q^{2}-2 q^{x+2}}{x(x+1)(x+2) q^{x+2}}\right\} .
\end{aligned}
$$

Let $\delta(x)=x^{2} p^{2}+x p^{2}-2 x p q+2 q^{2}-2 q^{x+2}$. We have

$$
\begin{aligned}
\delta(x+1)-\delta(x) & =2 x p^{2}+2 p^{2}-2 p q+2 q^{x+2}-2 q^{x+3} \\
& =2(x+1) p^{2}-2\left(q-q^{x+2}\right) \\
& =2(x+1) p^{2}-2 p^{2} \sum_{k=1}^{x+1} q^{k} \\
& >0,
\end{aligned}
$$

which implies that $\delta(x+1)>\delta(x)$. Because $\delta(1)=2 p^{3}>0$, by mathematical induction, we obtain $\delta(x)>0$ for every $x \in \mathbb{N}$. From this fact and (2.4), it follows that $\Delta^{2} g_{x_{0}}(x)>0$. Hence, the proof is complete.

Lemma 2.2. For $x_{0} \in \mathbb{N} \cup\{0\}$ and $x>0$, we have the following:

$$
\left|\Delta g_{x_{0}}(x)\right| \leq \begin{cases}\frac{1}{2} & \text { if } x_{0}=0 \\ \frac{1-q^{x_{0}+1}}{\left(x_{0}+1\right) p} \max \left\{p, \frac{1}{x_{0}+2}\right\} & \text { if } x_{0}>0\end{cases}
$$


Proof. It is clear that $\Delta g_{0}(x) \leq \frac{1}{2}$ for $x_{0}=0$. For $x \leq x_{0}$ and $x_{0}>0$, it follows from Lemma 2.1 that

$$
0<\Delta g_{x_{0}}(x) \leq \Delta g_{x_{0}}\left(x_{0}\right)=\frac{x_{0}-\sum_{k=1}^{x_{0}} q^{k}}{x_{0}\left(x_{0}+1\right)} \leq \frac{1-q^{x_{0}+1}}{x_{0}+1}
$$

and for $x>x_{0}>0$, we have

$$
0<-\Delta g_{x_{0}}(x)=\frac{\sum_{k=0}^{x_{0}} q^{k}}{x(x+1)} \leq \frac{\sum_{k=0}^{x_{0}} q^{k}}{\left(x_{0}+1\right)\left(x_{0}+2\right)} \leq \frac{1-q^{x_{0}+1}}{\left(x_{0}+1\right) p\left(x_{0}+2\right)}
$$

which yields $\left|\Delta g_{x_{0}}(x)\right| \leq \frac{1-q^{\mathrm{x}_{0}+1}}{\left(x_{0}+1\right) p} \max \left\{p, \frac{1}{x_{0}+2}\right\}$. Hence, (2.5) is obtained.

\section{Result}

The following theorem presents an improvement of the result in (1.2).

Theorem 3.1. For $x_{0} \in \mathcal{S}(x)$ and $\mu=\frac{q}{p}$, we have the following:

$$
\left|\mathbb{F}\left(x_{0}\right)-\mathbb{G}_{p}\left(x_{0}\right)\right| \leq E\left|(X+1) q-\sigma^{2} w(X) p\right| \Delta\left(x_{0}\right),
$$

where $\Delta(0)=\frac{1}{2}$ and $\Delta\left(x_{0}\right)=\frac{1-q^{\mathrm{x}_{0}+1}}{\left(x_{0}+1\right) p} \max \left\{p, \frac{1}{x_{0}+2}\right\}$ when $x_{0}>0$.

Proof. Using the same arguments detailed as in the proof of Theorem 2.1 in [2], we obtain

$$
\left|\mathbb{F}\left(x_{0}\right)-\mathbb{G}_{p}\left(x_{0}\right)\right| \leq E\left\{\left|(X+1) q-\sigma^{2} w(X) p \| \Delta g_{x_{0}}(X)\right|\right\} .
$$

Hence, with Lemma 2.2, the theorem is easily obtained.

The following corollary is a consequence of Theorem 3.1.

Corollary 3.1. If $(x+1) q / p-\sigma^{2} w(x)>/<0$ for every $x \in \mathcal{S}(x)$, then

$$
\left|\mathbb{F}\left(x_{0}\right)-\mathbb{G}_{p}\left(x_{0}\right)\right| \leq\left|\mu^{2}+\mu-\sigma^{2}\right| p \Delta\left(x_{0}\right),
$$

where $x_{0} \in \mathcal{S}(x)$.

Remark. The bound in Theorem 3.1 is sharper than the bound in (1.2) and is appropriate for any $q \in(0,1)$. 


\section{Examples}

We use the results in the Theorems 3.1 and Corollary 3.1 to give three examples concerning the beta-geometric, Pólya and Poisson distributions.

4.1. Let $X$ be the beta-geometric random variable with parameters $\alpha$ and $\beta$. Then its probability function is of the form

$$
p(x)=\frac{\alpha \Gamma(\beta+x) \Gamma(\alpha+\beta)}{\Gamma(\beta) \Gamma(\alpha+\beta+x+1)}, x=0,1, \ldots
$$

and the mean and variance of $X$ are $\mu=\frac{\beta}{\alpha-1}$ and $\sigma^{2}=\frac{\alpha \beta(\alpha+\beta-1)}{(\alpha-2)(\alpha-1)^{2}}$, respectively, where $\alpha>2$. Let $\mathbb{B} \mathbb{G}_{\alpha, \beta}\left(x_{0}\right)$ denote the beta-geometric distribution function at $x_{0} \in \mathbb{N} \cup\{0\}$. If $p=\frac{\alpha-1}{\alpha+\beta-1}$, then for $\alpha>2$, we have

$$
\left|\mathbb{B} \mathbb{G}_{\alpha, \beta}\left(x_{0}\right)-\mathbb{G}_{p}\left(x_{0}\right)\right| \leq \begin{cases}\frac{\beta}{(\alpha-2)(\alpha-1)} & \text { if } x_{0}=0 \\ \frac{2 \beta\left(1-q^{x_{0}+1}\right)}{(\alpha-2)(\alpha-1)\left(x_{0}+1\right) p} \max \left\{p, \frac{1}{x_{0}+2}\right\} & \text { if } x_{0}>0 .\end{cases}
$$

4.2. Let $X$ be the Pólya random variable with parameters $m$ and $d$. Then its probability function is of the form

$$
p(x)=\frac{\left(\begin{array}{c}
d+m-x-2 \\
m-x
\end{array}\right)}{\left(\begin{array}{c}
d+m-1 \\
m
\end{array}\right)}, x=0, \ldots, m .
$$

The mean and variance of $X$ are $\mu=\frac{m}{d}$ and $\sigma^{2}=\frac{m(d+m)(d-1)}{d^{2}(d+1)}$, respectively. Let $\mathbb{P Y}_{m, d}\left(x_{0}\right)$ denote the Pólya distribution function at $x_{0} \in\{0, \ldots, m\}$. If $p=\frac{d}{d+m}$, then

$$
\left|\mathbb{P Y}_{m, d}\left(x_{0}\right)-\mathbb{G}_{p}\left(x_{0}\right)\right| \leq \begin{cases}\frac{m}{d(d+1)} & \text { if } x_{0}=0, \\ \frac{2 m\left(1-q^{x_{0}+1}\right)}{d(d+1)\left(x_{0}+1\right) p} \max \left\{p, \frac{1}{x_{0}+2}\right\} & \text { if } x_{0}=1, \ldots, m .\end{cases}
$$

4.3. Let $X$ be the Poisson random variable with mean $\lambda$. Then its probability function is of the form

$$
p(x)=\frac{e^{-\lambda} \lambda^{x}}{x !}, x=0,1, \ldots
$$

The mean and variance of $X$ are $\mu=\sigma^{2}=\lambda$. Let $\mathbb{P}_{\lambda}\left(x_{0}\right)$ denote the Poisson distribution function at $x_{0} \in \mathbb{N} \cup\{0\}$. If $p=\frac{1}{1+\lambda}$, then

$$
\left|\mathbb{P}_{\lambda}\left(x_{0}\right)-\mathbb{G}_{p}\left(x_{0}\right)\right| \leq \begin{cases}\frac{2 \lambda^{2}}{(\lambda+1)} & \text { if } x_{0}=0 \\ \frac{\lambda^{2}\left(1-q^{x_{0}+1}\right)}{(\lambda+1)\left(x_{0}+1\right) p} \max \left\{p, \frac{1}{x_{0}+2}\right\} & \text { if } x_{0}>0\end{cases}
$$




\section{Conclusion}

The non-uniform bound in the Theorems 3.1, was improved by Stein's method and $w$-functions, provides a new general criteria for measuring the accuracy of the geometric approximation for non-negative integer-valued random variable. It is sharper than the bound in (1.2). In addition, this bound is more appropriate for any $q \in(0,1)$.

\section{References}

[1] M. Majsnerowska, A note on Poisson approximation by $w$-functions, Appl. Math., 25 (1998), 387-392.

[2] K. Teerapabolarn, Non uniform bounds on geometric approximation via Stein's method and $w$-functions, Comm. Statist. Theory Meth., 40 (2011), 145-158. 